\title{
SOME ESTIMATES ON THE HERMITE-HADAMARD INEQUALITY THROUGH GEOMETRICALLY QUASI-CONVEX FUNCTIONS
}

\author{
M. A. LATIF, SEVER S. DRAGOMIR, AND E. MOMONIAT \\ Received 20 October, 2015
}

\begin{abstract}
In present paper, by proving a new integral identity, using Hölder's inequality and mathematical analysis, we prove some Hermite-Hadamard type integral inequalities for geometrically quasi-convex functions which give better estimates to those already proven for the rightside of a Hermite-Hadamard type inequality established for geometrically convex functions in earlier works. A numerical example is also provided to support our claim. Applications of the results to special means of positive real numbers are given.
\end{abstract}

2010 Mathematics Subject Classification: 26D15; 26D20; 26D07

Keywords: Hermite-Hadamard's inequality, convex function, Quasi-Convex functions, geometrically quasi-convex function, Hölder integral inequality

\section{INTRODUCTION}

A function $g: J \subseteq \mathbb{R} \rightarrow \mathbb{R}$ is said to be convex on $J$ if

$$
g(\sigma l+(1-\sigma) m) \leq \sigma g(l)+(1-\sigma) g(m)
$$

holds for every $l, m \in J$ and $\sigma \in[0,1]$. There are several accomplishments of the role of convexity towards the field of inequalities and in the other branches of pure and applied mathematics but one of them is the celebrated Hermite Hadamard inequality, which is expressed as follows

$$
g\left(\frac{r+w}{2}\right) \leq \frac{1}{w-r} \int_{r}^{w} g(l) d l \leq \frac{g(r)+g(w)}{2},
$$

where $g: \varphi \neq J \subseteq \mathbb{R} \rightarrow \mathbb{R}$ is a convex function and $r, w \in J$ with $r<w$. Undeniably, the inequality (1.1) specifies a necessary and sufficient conditions for a function $g$ to be convex on the interval $[r, w]$.

The approach of quasi-convex functions speculates the concept of convex functions, that is a function $g: J \subseteq \mathbb{R} \rightarrow \mathbb{R}$ is quasi-convex on $J$ if the following relaxation of Jensen's inequality holds

$$
g(\sigma l+(1-\sigma) m) \leq \max \{g(l), g(m)\}
$$

(c) 2017 Miskolc University Press 
for $0 \leq \sigma \leq 1$.

A large number of papers have been written to provide refinements and generalizations of the inequalities (1.1) during the past few years by using convexity, quasiconvexity and their generalizations, see for instance [1-9], [11-17] and the references therein.

Many researchers have tried to generalize the notions of usual convexity and quasiconvexity in a number of approaches, one of the generalizations of the usual convexity is the geometric convexity which is avowed in the definition below.

Definition 1 ([10]). A function $g: J \subseteq(0, \infty) \rightarrow(0, \infty)$ is said to be geometrically convex on $J$ if

$$
g\left(l^{\sigma} m^{1-\sigma}\right) \leq[g(l)]^{\sigma}[g(m)]^{1-\sigma}
$$

holds for $x, y \in J$ and $\sigma \in[0,1]$.

The definition was further generalized by Qi and $\mathrm{Xi}$ in [12] as follows.

Definition 2 ([12]). A function $g: J \subseteq[0, \infty) \rightarrow[0, \infty)$ is called geometrically quasi-convex function on $J$ if

$$
g\left(l^{\sigma} m^{1-\sigma}\right) \leq \sup \{g(l), g(m)\}
$$

holds for $l, m \in J$ and $\sigma \in[0,1]$.

Remark 1. [12] If $g: J \subseteq[0, \infty) \rightarrow[0, \infty)$ is decreasing and geometrically quasiconvex on $J$, then it is quasi-convex on $J$. If $g: J \subseteq[0, \infty) \rightarrow[0, \infty)$ is increasing and quasi-convex on $J$, then it is geometrically quasi-convex on $J$.

Example 1. The function $g(l)=l^{s}, l \in \mathbb{R}_{+}$for $s \in \mathbb{R}$ geometrically quasi-convex on $\mathbb{R}_{+}$because for all $\sigma \in[0,1]$, the following inequality holds

$$
\left|g\left(l^{\sigma} m^{1-\sigma}\right)\right|^{s}=l^{s \sigma} m^{s(1-\sigma)} \leq\left\{\begin{array}{cc}
m^{s}, & s \geq 0 \\
l^{s}, & s<0 .
\end{array}\right.
$$

Some of the main results from [12] for geometrically quasi-convex functions are given in following theorems.

Theorem 1 ([12]). Let $g: J \subseteq \mathbb{R}_{+} \rightarrow \mathbb{R}$ be a differentiable on $J^{\circ}$ and $g^{\prime} \in$ $L([r, w])$ for $r, w \in J^{\circ}$ with $r<w$. If $\left|g^{\prime}\right|^{q}$ is a convex function on $[r, w]$, then

$$
\begin{aligned}
\mid \frac{(\ln w) g(w)-(\ln r) g(r)}{\ln w-\ln r}-\frac{1}{\ln w-\ln r} & \int_{r}^{w} \frac{g(l)}{l} d l \mid \\
& \leq N(r, w) \sup \left\{\left|g^{\prime}(r)\right|,\left|g^{\prime}(w)\right|\right\},
\end{aligned}
$$


where

$$
\begin{aligned}
& N(r, w)=\int_{0}^{1} r^{1-\sigma} w^{\sigma}\left|\ln \left(r^{1-\sigma} w^{\sigma}\right)\right| d \sigma \\
& = \begin{cases}\frac{w \ln w-r \ln r-(w-r)}{2(\ln w-\ln r)}, & r \geq 1, \\
\frac{w \ln w+r \ln r+2-w-r}{\ln w-\ln r}, & r<1<w, \\
\frac{w-r-(w \ln w-r \ln r)}{2(\ln w-\ln r)}, & w \leq 1 .\end{cases}
\end{aligned}
$$

Theorem 2 ([12]). Let $g: J \subseteq \mathbb{R}_{+} \rightarrow \mathbb{R}$ be a differentiable mapping on $J^{\circ}$ and $g^{\prime} \in L[r, w]$ for $r, w \in J^{\circ}$ with $r<w$. If $\left|g^{\prime}\right|^{q}$ is a geometrically quasi-convex on $[r, w]$ for $q>1$, then

$$
\begin{aligned}
& \left|\frac{(\ln w) g(w)-(\ln r) g(r)}{\ln w-\ln r}-\frac{1}{\ln w-\ln r} \int_{r}^{w} \frac{g(l)}{l} d l\right| \\
& \quad \leq\left\{\frac{q-1}{q} N\left(r^{\frac{q}{q-1}}, w^{\frac{q}{q-1}}\right)\right\}^{1-\frac{1}{q}}[M(r, w)]^{\frac{1}{q}} \sup \left\{\left|g^{\prime}(r)\right|,\left|g^{\prime}(w)\right|\right\},
\end{aligned}
$$

where $N(r, w)$ is defined in Theorem 1 and

$$
\begin{gathered}
M(r, w)=\int_{0}^{1}\left|\ln \left(r^{1-\sigma} w^{\sigma}\right)\right| d \sigma \\
= \begin{cases}\frac{w \ln w+r \ln r}{2}, & r \geq 1, \\
\frac{(\ln w)^{2}+(\ln r)^{2}}{\ln w-\ln r}, & r<1<w, \\
-\frac{w \ln w+r \ln r}{2}, & w \leq 1 .\end{cases}
\end{gathered}
$$

Theorem 3 ([12]). Let $g: J \subseteq \mathbb{R}_{+} \rightarrow \mathbb{R}$ be a differentiable on $J^{\circ}$ and $g^{\prime} \in L[r, w]$ for $r, w \in J^{\circ}$ with $r<w$. If $\left|g^{\prime}\right|^{q}$ is a geometrically convex on $[r, w]$ for $q>1$ and $\ell$ is a real number such that $q>\ell>0$. Then

$$
\begin{gathered}
\left|\frac{(\ln w) g(w)-(\ln r) g(r)}{\ln w-\ln r}-\frac{1}{\ln w-\ln r} \int_{r}^{w} \frac{g(l)}{l} d l\right| \leq\left(\frac{q-1}{q-\ell}\right)^{1-\frac{1}{q}}\left(\frac{1}{\ell}\right)^{\frac{1}{q}} \\
\times\left[N\left(r^{\frac{q-\ell}{q-1}}, w^{\frac{q-\ell}{q-1}}\right)\right]^{1-\frac{1}{q}}\left[N\left(r^{\ell}, w^{\ell}\right)\right]^{\frac{1}{q}} \sup \left\{\left|g^{\prime}(r)\right|,\left|g^{\prime}(w)\right|\right\},
\end{gathered}
$$

where $M(r, w)$ and $N(r, w)$ are defined in Theorem 1 and Theorem 2.

The main objective of the present paper is to provide new Hermite-Hadamard type inequalities for geometrically quasi-convex functions which provide refinements of 
the results given in [12]. A numerical example is provided to justify our claim. Applications of our results to special means are presented as well in Section 3.

\section{INEQUALITIES FOR GEOMETRICALLY QUASI-CONVEX FUNCTIONS}

The following two Lemmas are requisite to authorize our major determinations in this section:

Lemma 1. Suppose $J \subseteq \mathbb{R} \rightarrow \mathbb{R}$ be a differentiable on $J^{\circ}$ and $g^{\prime} \in L([r, w])$, for $r, w \in J^{\circ}$ and $r<w$, then

$$
\begin{aligned}
& \frac{(\ln w) g(w)-(\ln r) g(r)}{\ln w-}-\frac{1}{\ln r} \int_{r}^{w} \frac{g(l)}{l} d l \\
& =\frac{1}{2} \int_{0}^{1} r^{\frac{1-\sigma}{2}} w^{\frac{1+\sigma}{2}} \ln \left(r^{\frac{1-\sigma}{2}} w^{\frac{1+\sigma}{2}}\right) g^{\prime}\left(r^{\frac{1-\sigma}{2}} w^{\frac{1+\sigma}{2}}\right) d \sigma \\
& \quad+\frac{1}{2} \int_{0}^{1} r^{\frac{1+\sigma}{2}} w^{\frac{1-\sigma}{2}} \ln \left(r^{\frac{1+\sigma}{2}} w^{\frac{1-\sigma}{2}}\right) g^{\prime}\left(r^{\frac{1+\sigma}{2}} w^{\frac{1-\sigma}{2}}\right) d \sigma .
\end{aligned}
$$

Proof. Let

$$
J_{1}=\frac{1}{2} \int_{0}^{1} r^{\frac{1-\sigma}{2}} w^{\frac{1+\sigma}{2}} \ln \left(r^{\frac{1-\sigma}{2}} w^{\frac{1+\sigma}{2}}\right) g^{\prime}\left(r^{\frac{1-\sigma}{2}} w^{\frac{1+\sigma}{2}}\right) d \sigma
$$

and

$$
J_{2}=\frac{1}{2} \int_{0}^{1} r^{\frac{1+\sigma}{2}} w^{\frac{1-\sigma}{2}} \ln \left(r^{\frac{1+\sigma}{2}} w^{\frac{1-\sigma}{2}}\right) g^{\prime}\left(r^{\frac{1+\sigma}{2}} w^{\frac{1-\sigma}{2}}\right) d \sigma .
$$

Let $l=r^{\frac{1-\sigma}{2}} w^{\frac{1+\sigma}{2}}$ in $J_{1}$ and integrating by parts, we get

$$
\begin{aligned}
J_{1}= & \frac{1}{\ln w-\ln r} \int_{\sqrt{r w}}^{w}(\ln l) g^{\prime}(l) d l \\
& =\frac{1}{\ln w-\ln r}\left[(\ln w) g(w)-\ln (\sqrt{r w}) g(\sqrt{r w})-\int_{\sqrt{r w}}^{w} \frac{g(l)}{l} d l\right] .
\end{aligned}
$$

Now let $l=r^{\frac{1+\sigma}{2}} w^{\frac{1-\sigma}{2}}$ in $J_{2}$ and integrating by parts, we have

$$
\begin{aligned}
J_{2}= & \frac{1}{\ln w-\ln r} \int_{r}^{\sqrt{r w}}(\ln l) g^{\prime}(l) d l \\
& =\frac{1}{\ln w-\ln r}\left[\ln (\sqrt{r w}) g(\sqrt{r w})-(\ln r) g(r)-\int_{r}^{\sqrt{r w}} \frac{g(l)}{l} d l\right] .
\end{aligned}
$$

By adding (2.3) and (2.2), we get required result. 
Lemma 2. For $w>r>0$, we have

$$
\begin{aligned}
& \xi(r, w):=\int_{0}^{1}\left|\ln \left(r^{\frac{1-\sigma}{2}} w^{\frac{1+\sigma}{2}}\right)\right| d \sigma \\
& = \begin{cases}-\frac{\ln r+3 \ln w}{4}, & r<w<1, \\
\frac{\ln r+3 \ln w}{4}, & 1<r<w, \\
\frac{5(\ln w)^{2}+(\ln r)^{2}+2(\ln r)(\ln w)}{4(\ln w-\ln r)}, & r<1<w, r w<1, \\
\frac{(\ln w)^{2}}{\ln w-\ln r}, & r<1<w, r w=1\end{cases}
\end{aligned}
$$

and

$$
\begin{aligned}
& \eta(r, w):=\int_{0}^{1} r^{\frac{1-\sigma}{2}} w^{\frac{1+\sigma}{2}}\left|\ln \left(r^{\frac{1-\sigma}{2}} w^{\frac{1+\sigma}{2}}\right)\right| d \sigma \\
& = \begin{cases}\frac{2[w-\sqrt{r w}+\sqrt{r w} \ln (\sqrt{r w})-w \ln w]}{\ln w-\ln r}, & r<w<1, \\
\frac{2[-w+\sqrt{r w}-\sqrt{r w} \ln (\sqrt{r w})+w \ln w]}{\ln w-\ln r}, & 1<r<w, \\
\frac{2[2+\sqrt{r w} \ln (\sqrt{r w})-\sqrt{r w}+w \ln w-w]}{\ln w-\ln r}, & r<1<w, r w<1, \\
\frac{2[w \ln w-w+1]}{\ln w-\ln r}, & r<1<w, r w=1 .\end{cases}
\end{aligned}
$$

Proof. The proof can be done by simple computations.

Theorem 4. Let $g: J \subseteq \mathbb{R}_{+} \rightarrow \mathbb{R}$ be a differentiable mapping on $J^{\circ}$ and $g^{\prime} \in$ $L[r, w]$ for $r, w \in J^{\circ}$ with $r<w$. If $\left|g^{\prime}\right|$ is a geometrically quasi-convex on $[r, w]$, then

$$
\begin{aligned}
& \left|\frac{(\ln w) g(w)-(\ln r) g(r)}{\ln w-\ln r}-\frac{1}{\ln w-\ln r} \int_{r}^{w} \frac{g(l)}{l} d l\right| \\
& \leq \frac{\eta(r, w)}{2}\left(\sup \left\{\left|g^{\prime}(\sqrt{r w})\right|,\left|g^{\prime}(w)\right|\right\}\right) \\
& \quad+\frac{\eta(w, r)}{2}\left(\sup \left\{\left|g^{\prime}(r)\right|,\left|g^{\prime}(\sqrt{r w})\right|\right\}\right),
\end{aligned}
$$

where $\eta(r, w)$ is defined in Lemma 2.

Proof. From Lemma 1 and using Hölder inequality, It is easy to observe that the following inequality holds 


$$
\begin{aligned}
& \left|\frac{(\ln w) g(w)-(\ln r) g(r)}{\ln w-\ln r}-\frac{1}{\ln w-\ln r} \int_{r}^{w} \frac{g(l)}{l} d l\right| \\
& \leq \frac{1}{2} \int_{0}^{1} r^{\frac{1-\sigma}{2}} w^{\frac{1+\sigma}{2}}\left|\ln \left(r^{\frac{1-\sigma}{2}} w^{\frac{1+\sigma}{2}}\right)\right|\left|g^{\prime}\left(r^{\frac{1-\sigma}{2}} w^{\frac{1+\sigma}{2}}\right)\right| d \sigma \\
& \quad+\frac{1}{2} \int_{0}^{1} r^{\frac{1+\sigma}{2}} w^{\frac{1-\sigma}{2}}\left|\ln \left(r^{\frac{1+\sigma}{2}} w^{\frac{1-\sigma}{2}}\right)\right|\left|g^{\prime}\left(r^{\frac{1+\sigma}{2}} w^{\frac{1-\sigma}{2}}\right)\right| d \sigma
\end{aligned}
$$

By using the geometric quasi-convexity of $\left|g^{\prime}\right|$ on $[r, w]$, we have

$$
\left|g^{\prime}\left(r^{\frac{1-\sigma}{2}} w^{\frac{1+\sigma}{2}}\right)\right| \leq \sup \left\{\left|g^{\prime}(\sqrt{r w})\right|,\left|g^{\prime}(w)\right|\right\}
$$

and

$$
\left|g^{\prime}\left(r^{\frac{1+\sigma}{2}} w^{\frac{1-\sigma}{2}}\right)\right| \leq \sup \left\{\left|g^{\prime}(r)\right|,\left|g^{\prime}(\sqrt{r w})\right|\right\} .
$$

By using Lemma 2 and the above results, we obtain

$$
\begin{array}{r}
\int_{0}^{1} r^{\frac{1-\sigma}{2}} w^{\frac{1+\sigma}{2}}\left|\ln \left(r^{\frac{1-\sigma}{2}} w^{\frac{1+\sigma}{2}}\right)\right|\left|g^{\prime}\left(r^{\frac{1-\sigma}{2}} w^{\frac{1+\sigma}{2}}\right)\right| d \sigma \\
\leq \sup \left\{\left|g^{\prime}(\sqrt{r w})\right|,\left|g^{\prime}(w)\right|\right\} \int_{0}^{1} r^{\frac{1-\sigma}{2}} w^{\frac{1+\sigma}{2}}\left|\ln \left(r^{\frac{1-\sigma}{2}} w^{\frac{1+\sigma}{2}}\right)\right| d \sigma \\
=\eta(r, w)\left(\sup \left\{\left|g^{\prime}(\sqrt{r w})\right|,\left|g^{\prime}(w)\right|\right\}\right)
\end{array}
$$

and

$$
\begin{array}{r}
\int_{0}^{1} r^{\frac{1+\sigma}{2}} w^{\frac{1-\sigma}{2}}\left|\ln \left(r^{\frac{1+\sigma}{2}} w^{\frac{1-\sigma}{2}}\right)\right|\left|g^{\prime}\left(r^{\frac{1+\sigma}{2}} w^{\frac{1-\sigma}{2}}\right)\right| d \sigma \\
\leq \sup \left\{\left|g^{\prime}(r)\right|,\left|g^{\prime}(\sqrt{r w})\right|\right\} \int_{0}^{1} r^{\frac{1+\sigma}{2}} w^{\frac{1-\sigma}{2}}\left|\ln \left(r^{\frac{1+\sigma}{2}} w^{\frac{1-\sigma}{2}}\right)\right| d \sigma \\
=\eta(w, r)\left(\sup \left\{\left|g^{\prime}(r)\right|,\left|g^{\prime}(\sqrt{r w})\right|\right\}\right) .
\end{array}
$$

The inequality (2.4) can be obtained by using (2.6) and (2.7) in (2.5).

Theorem 5. Let $g: J \subseteq \mathbb{R}_{+} \rightarrow \mathbb{R}$ be a differentiable mapping on $J^{\circ}$ and $g^{\prime} \in$ $L[r, w]$ for $r, w \in J^{\circ}$ with $r<w$. If $\left|g^{\prime}\right|^{q}$ is a geometrically quasi-convex on $[r, w]$ for $q>1$, then

$$
\begin{aligned}
& \left|\frac{(\ln w) g(w)-(\ln r) g(r)}{\ln w-\ln r}-\frac{1}{\ln w-\ln r} \int_{r}^{w} \frac{g(l)}{l} d l\right| \\
& \leq \frac{1}{2}\left\{\frac{q-1}{q} \eta\left(r^{\frac{q}{q-1}}, w^{\frac{q}{q-1}}\right)\right\}^{1-\frac{1}{q}}\left[\xi(r, w)\left(\sup \left\{\left|g^{\prime}(\sqrt{r w})\right|^{q},\left|g^{\prime}(w)\right|^{q}\right\}\right)\right]^{\frac{1}{q}}
\end{aligned}
$$




$$
+\frac{1}{2}\left\{\frac{q-1}{q} \eta\left(w^{\frac{q}{q-1}}, r^{\frac{q}{q-1}}\right)\right\}^{1-\frac{1}{q}}\left[\xi(w, r)\left(\sup \left\{\left|g^{\prime}(w)\right|^{q},\left|g^{\prime}(\sqrt{r w})\right|^{q}\right\}\right)\right]^{\frac{1}{q}},
$$

where $\xi(r, w)$ and $\eta(r, w)$ are defined in Lemma 2.

Proof. From Lemma 1 and using Hölder inequality, we have

$$
\begin{aligned}
& \mid \frac{(\ln w) g(w)}{\ln w}-\ln r\ln r) g(r) \\
& \leq \frac{1}{2} \int_{0}^{1} r^{\frac{1-\sigma}{2}} w^{\frac{1+\sigma}{2}}\left|\ln \left(r^{\frac{1-\sigma}{2}} w^{\frac{1+\sigma}{2}}\right)\right|\left|g^{\prime}\left(r^{\frac{1-\sigma}{2}} w^{\frac{1+\sigma}{2}}\right)\right| d \sigma \\
&+ \frac{1}{2} \int_{0}^{1} r^{\frac{1+\sigma}{2}} w^{\frac{1-\sigma}{2}}\left|\ln \left(r^{\frac{1+\sigma}{2}} w^{\frac{1-\sigma}{2}}\right)\right|\left|g^{\prime}\left(r^{\frac{1+\sigma}{2}} w^{\frac{1-\sigma}{2}}\right)\right| d \sigma \\
& \leq \frac{1}{2}\left(\int_{0}^{1} r^{\frac{q(1-\sigma)}{2(q-1)}} w^{\frac{q(1+\sigma)}{2(q-1)}}\left|\ln \left(r^{\frac{1-\sigma}{2}} w^{\frac{1+\sigma}{2}}\right)\right| d \sigma\right)^{1-\frac{1}{q}} \\
& \times\left(\int_{0}^{1}\left|\ln \left(r^{\frac{1-\sigma}{2}} w^{\frac{1+\sigma}{2}}\right)\right|\left|g^{\prime}\left(r^{\frac{1-\sigma}{2}} w^{\frac{1+\sigma}{2}}\right)\right|^{q} d \sigma\right)^{\frac{1}{q}} \\
&+ \frac{1}{2}\left(\int_{0}^{1} r^{\frac{q(1+\sigma)}{2(q-1)}} w^{\frac{q(1-\sigma)}{2(q-1)}}\left|\ln \left(r^{\frac{1+\sigma}{2}} w^{\frac{1-\sigma}{2}}\right)\right| d \sigma\right)^{1-\frac{1}{q}} \\
& \times\left(\int_{0}^{1}\left|\ln \left(r^{\frac{1+\sigma}{2}} w^{\frac{1-\sigma}{2}}\right)\right|\left|g^{\prime}\left(r^{\frac{1+\sigma}{2}} w^{\frac{1-\sigma}{2}}\right)\right|^{q} d \sigma\right)^{\frac{1}{q}} .
\end{aligned}
$$

By using the geometric quasi-convexity of $\left|g^{\prime}\right|^{q}$ on $[r, w]$, we obtain

$$
\begin{aligned}
\int_{0}^{1}\left|\ln \left(r^{\frac{1-\sigma}{2}} w^{\frac{1+\sigma}{2}}\right)\right|\left|g^{\prime}\left(r^{\frac{1-\sigma}{2}} w^{\frac{1+\sigma}{2}}\right)\right|^{q} d \sigma & \\
\leq\left(\operatorname { s u p } \left\{\left|g^{\prime}(\sqrt{r w})\right|^{q}\right.\right. & \left.\left.,\left|g^{\prime}(w)\right|^{q}\right\}\right) \int_{0}^{1}\left|\ln \left(r^{\frac{1-\sigma}{2}} w^{\frac{1+\sigma}{2}}\right)\right| d \sigma \\
& =\xi(r, w)\left(\sup \left\{\left|g^{\prime}(\sqrt{r w})\right|^{q},\left|g^{\prime}(w)\right|^{q}\right\}\right)
\end{aligned}
$$

and

$$
\begin{aligned}
& \int_{0}^{1}\left|\ln \left(r^{\frac{1+\sigma}{2}} w^{\frac{1-\sigma}{2}}\right)\right|\left|g^{\prime}\left(r^{\frac{1+\sigma}{2}} w^{\frac{1-\sigma}{2}}\right)\right|^{q} d \sigma \\
& \leq\left(\sup \left\{\left|g^{\prime}(r)\right|^{q},\left|g^{\prime}(\sqrt{r w})\right|^{q}\right\}\right) \int_{0}^{1}\left|\ln \left(r^{\frac{1+\sigma}{2}} w^{\frac{1-\sigma}{2}}\right)\right| d \sigma \\
& =\xi(w, r)\left(\sup \left\{\left|g^{\prime}(r)\right|^{q},\left|g^{\prime}(\sqrt{r w})\right|^{q}\right\}\right) .
\end{aligned}
$$


Now an application of Lemma 2, gives us the following results

$$
\begin{gathered}
\int_{0}^{1} r^{\frac{q(1-\sigma)}{2(q-1)}} w^{\frac{q(1+\sigma)}{2(q-1)}}\left|\ln \left(r^{\frac{1+\sigma}{2}} w^{\frac{1-\sigma}{2}}\right)\right| d \sigma=\frac{q-1}{q} \eta\left(r^{\frac{q}{q-1}}, w^{\frac{q}{q-1}}\right) \\
\int_{0}^{1} r^{\frac{q(1+\sigma)}{2(q-1)}} w^{\frac{q(1-\sigma)}{2(q-1)}}\left|\ln \left(r^{\frac{1-\sigma}{2}} w^{\frac{1+\sigma}{2}}\right)\right| d \sigma=\frac{q-1}{q} \eta\left(w^{\frac{q}{q-1}}, r^{\frac{q}{q-1}}\right), \\
\int_{0}^{1}\left|\ln \left(r^{\frac{1-\sigma}{2}} w^{\frac{1+\sigma}{2}}\right)\right| d \sigma=\xi(r, w)
\end{gathered}
$$

and

$$
\int_{0}^{1}\left|\ln \left(r^{\frac{1+\sigma}{2}} w^{\frac{1-\sigma}{2}}\right)\right| d \sigma=\xi(w, r) .
$$

A combination of the results (2.9)-(2.15) gives us the required inequality (2.8). Hence the proof of the theorem is complete.

Theorem 6. Let $g: J \subseteq \mathbb{R}_{+} \rightarrow \mathbb{R}$ be a differentiable on $J^{\circ}$ and $g^{\prime} \in L[r, w]$ for $r, w \in J^{\circ}$ with $r<w$. If $\left|g^{\prime}\right|^{q}$ is a geometrically convex on $[r, w]$ for $q>1$ and $\ell$ is $a$ real number such that $q>\ell>0$. Then

$$
\begin{aligned}
& \left|\frac{(\ln w) g(w)-(\ln r) g(r)}{\ln w-\ln r}-\frac{1}{\ln w-\ln r} \int_{r}^{w} \frac{g(l)}{l} d l\right| \leq \frac{1}{2}\left(\frac{q-1}{q-\ell}\right)^{1-\frac{1}{q}}\left(\frac{1}{\ell}\right)^{\frac{1}{q}} \\
& \quad \times\left\{\left[\eta\left(r^{\frac{q-\ell}{q-1}}, w^{\frac{q-\ell}{q-1}}\right)\right]^{1-\frac{1}{q}}\left[\eta\left(r^{\ell}, w^{\ell}\right)\left(\sup \left\{\left|g^{\prime}(\sqrt{r w})\right|^{q},\left|g^{\prime}(w)\right|^{q}\right\}\right)\right]^{\frac{1}{q}}\right. \\
& \left.+\left[\eta\left(w^{\frac{q-\ell}{q-1}}, r^{\frac{q-\ell}{q-1}}\right)\right]^{1-\frac{1}{q}}\left[\eta\left(w^{\ell}, r^{\ell}\right)\left(\sup \left\{\left|g^{\prime}(r)\right|^{q},\left|g^{\prime}(\sqrt{r w})\right|^{q}\right\}\right)\right]^{\frac{1}{q}}\right\},
\end{aligned}
$$

where $\eta(r, w)$ is defined in Lemma 2.

Proof.

$$
\begin{aligned}
& \left|\frac{(\ln w) g(w)-(\ln r) g(r)}{\ln w-\ln r}-\frac{1}{\ln w-\ln r} \int_{r}^{w} \frac{g(l)}{l} d l\right| \\
& \quad \leq \frac{1}{2} \int_{0}^{1} r^{\frac{1-\sigma}{2}} w^{\frac{1+\sigma}{2}}\left|\ln \left(r^{\frac{1-\sigma}{2}} w^{\frac{1+\sigma}{2}}\right)\right|\left|g^{\prime}\left(r^{\frac{1-\sigma}{2}} w^{\frac{1+\sigma}{2}}\right)\right| d \sigma \\
& \quad+\frac{1}{2} \int_{0}^{1} r^{\frac{1+\sigma}{2}} w^{\frac{1-\sigma}{2}}\left|\ln \left(r^{\frac{1+\sigma}{2}} w^{\frac{1-\sigma}{2}}\right)\right|\left|g^{\prime}\left(r^{\frac{1+\sigma}{2}} w^{\frac{1-\sigma}{2}}\right)\right| d \sigma \\
& \quad \leq \frac{1}{2}\left(\int_{0}^{1} r^{\frac{(q-\ell)(1-\sigma)}{2(q-1)}} w^{\frac{(q-\ell)(1+\sigma)}{2(q-1)}}\left|\ln \left(r^{\frac{1-\sigma}{2}} w^{\frac{1+\sigma}{2}}\right)\right| d \sigma\right)^{1-\frac{1}{q}} \\
& \times\left(\int_{0}^{1} r^{\frac{\ell(1-\sigma)}{2}} w^{\frac{\ell(1+\sigma)}{2}}\left|\ln \left(r^{\frac{1-\sigma}{2}} w^{\frac{1+\sigma}{2}}\right)\right|\left|g^{\prime}\left(r^{\frac{1-\sigma}{2}} w^{\frac{1+\sigma}{2}}\right)\right|^{q} d \sigma\right)^{\frac{1}{q}}
\end{aligned}
$$




$$
\begin{gathered}
+\frac{1}{2}\left(\int_{0}^{1} r^{\frac{(q-\ell)(1+\sigma)}{2(q-1)}} w^{\frac{(q-\ell)(1-\sigma)}{2(q-1)}}\left|\ln \left(r^{\frac{1+\sigma}{2}} w^{\frac{1-\sigma}{2}}\right)\right| d \sigma\right)^{1-\frac{1}{q}} \\
\times\left(\int_{0}^{1} r^{\frac{\ell(1+\sigma)}{2}} w^{\frac{\ell(1-\sigma)}{2}}\left|\ln \left(r^{\frac{1+\sigma}{2}} w^{\frac{1-\sigma}{2}}\right)\right|\left|g^{\prime}\left(r^{\frac{1+\sigma}{2}} w^{\frac{1-\sigma}{2}}\right)\right|^{q} d \sigma\right)^{\frac{1}{q}} .
\end{gathered}
$$

Now by using the quasi-geometric convexity of $\left|g^{\prime}\right|^{q}$ on $[r, w]$ and Lemma 2, we have

$$
\begin{aligned}
& \int_{0}^{1} r^{\frac{\ell(1-\sigma)}{2}} w^{\frac{\ell(1+\sigma)}{2}}\left|\ln \left(r^{\frac{1-\sigma}{2}} w^{\frac{1+\sigma}{2}}\right)\right|\left|g^{\prime}\left(r^{\frac{1-\sigma}{2}} w^{\frac{1+\sigma}{2}}\right)\right|^{q} d \sigma \\
& \leq \frac{1}{\ell} \eta\left(r^{\ell}, w^{\ell}\right)\left(\sup \left\{\left|g^{\prime}(\sqrt{r w})\right|^{q},\left|g^{\prime}(w)\right|^{q}\right\}\right), \\
& \int_{0}^{1} r^{\frac{\ell(1+\sigma)}{2}} w^{\frac{\ell(1-\sigma)}{2}}\left|\ln \left(r^{\frac{1+\sigma}{2}} w^{\frac{1-\sigma}{2}}\right)\right|\left|g^{\prime}\left(r^{\frac{1+\sigma}{2}} w^{\frac{1-\sigma}{2}}\right)\right|^{q} d \sigma \\
& \leq \frac{1}{\ell} \eta\left(w^{\ell}, r^{\ell}\right)\left(\sup \left\{\left|g^{\prime}(r)\right|^{q},\left|g^{\prime}(\sqrt{r w})\right|^{q}\right\}\right), \\
& \int_{0}^{1} r^{\frac{(q-\ell)(1-\sigma)}{2(q-1)}} w^{\frac{(q-\ell)(1+\sigma)}{2(q-1)}}\left|\ln \left(r^{\frac{1-\sigma}{2}} w^{\frac{1+\sigma}{2}}\right)\right| d \sigma \\
& =\left(\frac{q-1}{q-\ell}\right) \eta\left(r^{\frac{q-\ell}{q-1}}, w^{\frac{q-\ell}{q-1}}\right)
\end{aligned}
$$

and

$$
\begin{aligned}
& \int_{0}^{1} r^{\frac{(q-\ell)(1+\sigma)}{2(q-1)}} w^{\frac{(q-\ell)(1-\sigma)}{2(q-1)}}\left|\ln \left(r^{\frac{1+\sigma}{2}} w^{\frac{1-\sigma}{2}}\right)\right| d \sigma \\
& =\left(\frac{q-1}{q-\ell}\right) \eta\left(w^{\frac{q-\ell}{q-1}}, r^{\frac{q-\ell}{q-1}}\right) .
\end{aligned}
$$

Applying (2.18)-(2.21) in (2.17), we get (2.16). This completes the proof of the theorem.

Theorem 7. Let $g: \mathbb{R}_{+} \rightarrow \mathbb{R}_{0}$ be a geometrically quasi-convex function on $[r, w]$ and $g \in L([r, w])$. Then

$$
\begin{aligned}
g\left((r w)^{\frac{1}{2}}\right) \leq \frac{1}{\ln w-} & \ln r \\
\leq & \int_{r}^{w} \frac{g(l)}{l} d l \\
\leq & \sup \{g(r), g(\sqrt{r w})\}+\sup \{g(\sqrt{r w}), g(w)\}] .
\end{aligned}
$$

Proof. It is easy to observe that

$$
(r w)^{\frac{1}{2}}=r^{\frac{1}{2}\left(\frac{1-\sigma}{2}\right)} w^{\frac{1}{2}\left(\frac{1+\sigma}{2}\right)} r^{\frac{1}{2}\left(\frac{1+\sigma}{2}\right)} w^{\frac{1}{2}\left(\frac{1-\sigma}{2}\right)}
$$


for $0 \leq \sigma \leq 1$. Since $g(l)$ is geometrically quasi- convex on $[r, w]$, we have

$$
g\left((r w)^{\frac{1}{2}}\right) \leq \sup \left\{g\left(r^{\frac{1-\sigma}{2}} w^{\frac{1+\sigma}{2}}\right), g\left(r^{\frac{1+\sigma}{2}} w^{\frac{1-\sigma}{2}}\right)\right\} .
$$

Now we consider the two cases:

Case I

If

then

$$
\sup \left\{g\left(r^{\frac{1-\sigma}{2}} w^{\frac{1+\sigma}{2}}\right), g\left(r^{\frac{1+\sigma}{2}} w^{\frac{1-\sigma}{2}}\right)\right\}=g\left(r^{\frac{1-\sigma}{2}} w^{\frac{1+\sigma}{2}}\right)
$$

$$
g\left((r w)^{\frac{1}{2}}\right) \leq g\left(r^{\frac{1-\sigma}{2}} w^{\frac{1+\sigma}{2}}\right) .
$$

Integrating (2.24) with respect to $\sigma$ over $[0,1]$, we obtain

$$
\begin{aligned}
g\left((r w)^{\frac{1}{2}}\right) \leq \frac{2}{\ln w-\ln r} \int_{\sqrt{r w}}^{w} \frac{g(l)}{l} d l=\int_{0}^{1} g\left(r^{\frac{1-\sigma}{2}} w^{\frac{1+\sigma}{2}}\right) d \sigma & \leq \sup \{g(\sqrt{r w}), g(w)\} . \\
\int_{0}^{1} g\left(r^{\frac{1-\sigma}{2}} w^{\frac{1+\sigma}{2}}\right) d \sigma=\int_{0}^{1} g\left(r^{\frac{1+\sigma}{2}} w^{\frac{1-\sigma}{2}}\right) d \sigma & =\frac{1}{\ln w-\ln r} \int_{r}^{w} \frac{g(l)}{l} d l
\end{aligned}
$$

Case II

If

$$
\sup \left\{g\left(r^{\frac{1-\sigma}{2}} w^{\frac{1+\sigma}{2}}\right), g\left(r^{\frac{1+\sigma}{2}} w^{\frac{1-\sigma}{2}}\right)\right\}=g\left(r^{\frac{1+\sigma}{2}} w^{\frac{1+\sigma}{2}}\right)
$$

then

$$
g\left((r w)^{\frac{1}{2}}\right) \leq g\left(r^{\frac{1+\sigma}{2}} w^{\frac{1-\sigma}{2}}\right) .
$$

Integrating (2.27) with respect to $\sigma$ over $[0,1]$, we get

$$
\begin{array}{rl}
g\left((r w)^{\frac{1}{2}}\right) \leq \frac{2}{\ln w-\ln r} \int_{r}^{\sqrt{r w}} \frac{g(l)}{l} d l=\int_{0}^{1} & g\left(r^{\frac{1+\sigma}{2}} w^{\frac{1-\sigma}{2}}\right) d \sigma \\
& \leq \sup \{g(r), g(\sqrt{r w})\} .
\end{array}
$$

Adding (2.25) and (2.28) and dividing the resulting inequality by 2 , we get the inequality (2.22). Thus the proof of the theorem is completed.

Theorem 8. Let $g, h:[r, w] \subseteq \mathbb{R} \rightarrow \mathbb{R}$ be a geometrically convex function on $[r, w]$ and $g h \in L([r, w])$, then the following inequality holds

$$
\begin{aligned}
& \frac{1}{\ln w-\ln r} \int_{r}^{w} \frac{g(l)}{l} h(l) d l \\
& \leq \frac{1}{2}[\sup \{g(\sqrt{r w}) h(\sqrt{r w}), g(\sqrt{r w}) h(w), g(w) h(\sqrt{r w}), g(w) h(w)\} \\
& +\sup \{g(\sqrt{r w}) h(\sqrt{r w}), g(r) h(\sqrt{r w}), g(\sqrt{r w}) h(r), g(r) h(r)\}] \text {. }
\end{aligned}
$$


Proof. Let $l=r^{\frac{1-\sigma}{2}} w^{\frac{1+\sigma}{2}}, 0 \leq \sigma \leq 1$ and using the geometric quasi-convex of $g$ and $h$ on $[r, w]$, we have

$$
\begin{aligned}
& \frac{2}{\ln w-\ln r} \int_{\sqrt{r w}}^{w} \frac{g(l) h(l)}{l} d l=\int_{0}^{1} g\left(r^{\frac{1-\sigma}{2}} w^{\frac{1+\sigma}{2}}\right) h\left(r^{\frac{1-\sigma}{2}} w^{\frac{1+\sigma}{2}}\right) d \sigma \\
& \leq \sup \{g(\sqrt{r w}) h(\sqrt{r w}), g(w) h(\sqrt{r w}), g(\sqrt{r w}) h(w), g(w) h(w)\} .
\end{aligned}
$$

Similarly, the substitution $l=r^{\frac{1+\sigma}{2}} w^{\frac{1-\sigma}{2}}, 0 \leq \sigma \leq 1$, gives us the following result

$$
\begin{aligned}
& \frac{2}{\ln w-\ln r} \int_{r}^{\sqrt{r w}} \frac{g(l) h(l)}{l} d l=\int_{0}^{1} g\left(r^{\frac{1-\sigma}{2}} w^{\frac{1+\sigma}{2}}\right) h\left(r^{\frac{1-\sigma}{2}} w^{\frac{1+\sigma}{2}}\right) d \sigma \\
& \quad \leq \sup \{g(\sqrt{r w}) h(\sqrt{r w}), g(r) h(\sqrt{r w}), g(\sqrt{r w}) h(r), g(r) h(r)\} .
\end{aligned}
$$

Adding (2.30) and (2.31) and dividing the resulting inequality by 2 , we get (2.29).

\section{Applications to SPECial MEANS}

Let $w>r>0$ and $s \in \mathbb{R}$. Consider the following means

$$
\begin{gathered}
G(r, w)=\sqrt{r w}, \\
I(r, w)= \begin{cases}\frac{1}{e}\left(\frac{w^{w}}{r^{r}}\right)^{\frac{1}{w-r}}, & r \neq w, \\
a, & r=w,\end{cases} \\
L(a, b)= \begin{cases}\frac{w-r}{\ln w-\ln r}, & r \neq w, \\
r, & r=w,\end{cases}
\end{gathered}
$$

and

$$
L_{s}(r, w)= \begin{cases}{\left[\frac{w^{s+1}+r^{s+1}}{(s+1)(w-r)}\right]^{\frac{1}{s}},} & s \neq-1,0, \\ L(r, w), & s=-1, \\ I(r, w), & s=0,\end{cases}
$$

The above means are known as respectively the geometric, the exponential, the logarithmic and the generalized logarithmic means of two positive real numbers $a$ and $b$.

We now give applications of our results to the above means. 
Theorem 9. Let $w>r>0$ and $s \in \mathbb{R}$.

(1) If $r<w<1$ and $s>0$, then

$$
\begin{aligned}
\ln I\left(r^{s+1}, w^{s+1}\right) \leq & \frac{|s+1| \ln I(G(a, b), w)}{(\ln w-\ln r)\left[L_{s}\left(r^{s+1}, w^{s+1}\right)\right]^{s}} \\
& \quad \times\left\{w^{s}(G(a, b)-w)+[G(a, b)]^{s}(G(a, b)-r)\right\} .
\end{aligned}
$$

(2) If $1<r<w$ and $s<-1$, then

$$
\begin{aligned}
\ln I\left(r^{s+1}, w^{s+1}\right) \leq- & \frac{|s+1| \ln I(G(a, b), w)}{(\ln w-\ln r)\left[L_{s}\left(r^{s+1}, w^{s+1}\right)\right]^{s}} \\
& \times\left\{[G(a, b)]^{s}(G(a, b)-w)+r^{s}(G(a, b)-r)\right\} .
\end{aligned}
$$

Proof. Let $g(l)=l^{s+1}, l \in \mathbb{R}_{+}$and $s \in \mathbb{R}, s \neq-1$. If $m>l>0$, then

$$
\left|g^{\prime}\left(l^{\frac{\sigma-1}{2}} m^{\frac{\sigma+1}{2}}\right)\right|^{s} \leq\left\{\begin{array}{cl}
|s+1| m^{s}, & , s \geq 0 \\
|s+1|(l m)^{\frac{s}{2}}, & s<0 .
\end{array}\right.
$$

Also

$$
\left|g^{\prime}\left(l^{\frac{\sigma+1}{2}} m^{\frac{\sigma-1}{2}}\right)\right|^{s} \leq\left\{\begin{array}{cl}
|s+1|(l m)^{\frac{s}{2}}, & , s \geq 0 \\
|s+1| l^{s}, & s<0 .
\end{array}\right.
$$

This shows that the function $\left|g^{\prime}(l)\right|=|s+1| l^{s}$ for $s \in \mathbb{R}, s \neq-1$, is geometrically quasi-convex on $\mathbb{R}_{+}$.

Now

$$
\begin{aligned}
& \frac{(\ln w) g(w)-(\ln r) g(r)}{\ln w-\ln r}-\frac{1}{\ln w-\ln r} \int_{r}^{w} \frac{g(l)}{l} d l \\
&= {\left[L_{s}\left(r^{s+1}, w^{s+1}\right)\right]^{s} \ln I\left(r^{s+1}, w^{s+1}\right), } \\
& \frac{1}{2} \eta(r, w)\left(\sup \left\{\left|g^{\prime}(\sqrt{r w})\right|,\left|g^{\prime}(w)\right|\right\}\right) \\
&=\frac{|s+1| w^{s}[w-\sqrt{r w}+\sqrt{r w} \ln (\sqrt{r w})-w \ln w]}{\ln w-\ln r} \\
&=\frac{|s+1| w^{s}(\sqrt{r w}-w) \ln I(\sqrt{r w}, w)}{\ln w-\ln r}
\end{aligned}
$$

and

$$
\begin{aligned}
& \frac{1}{2} \eta(w, r)\left(\sup \left\{\left|g^{\prime}(r)\right|,\left|g^{\prime}(\sqrt{r w})\right|\right\}\right) \\
& =\frac{|s+1|(r w)^{\frac{s}{2}}[r-\sqrt{r w}+\sqrt{r w} \ln (\sqrt{r w})-r \ln r]}{\ln w-\ln r}
\end{aligned}
$$




$$
=\frac{|s+1|(r w)^{\frac{s}{2}}(\sqrt{r w}-r) \ln I(\sqrt{r w}, r)}{\ln w-\ln r} .
$$

Substituting (3.3), (3.4) and (3.5) in Theorem 4, we get (3.1). The inequality (3.2) can be obtained in similar way.

The following numerical example illustrates that our results provide refinements of the results given in [12].

For the geometrically quasi-convex function $g(x)=x^{3}, x \in \mathbb{R}_{+}$, the following table is prepared using mathematica.

\begin{tabular}{|c|c|c|}
\hline Function & Error Bound of Theorem 4 & Error Bound of Theorem 1 \\
\hline$g(x)=x^{3}, a=\frac{1}{2}, b=\frac{2}{3}$ & 0.363473 & 0.418996 \\
\hline$g(x)=x^{3}, a=\frac{3}{2}, b=2$ & 10.4408 & 11.6002 \\
\hline$g(x)=x^{3}, a=\frac{1}{2}, b=\frac{3}{2}$ & 0.389078 & 1.60745 \\
\hline
\end{tabular}

Similarly we can make tables for comparison of the results given in Theorem 2 and Theorem 3 with those given in Theorem 5 and Theorem 6 respectively.

\section{ACKNOWLEDGEMENT}

The authors express their gratitude to the referees for their valuable comments which have been implemented in the final version of the manuscript.

\section{REFERENCES}

[1] M. Alomari, M. Darus, and S. Dragomir, "New inequalities of Hermite-Hadamard's type for functions whose second derivatives absolute values are quasi-convex," Tamk. J. Math, vol. 41, pp. 353-359, 2010.

[2] M. Alomari, M. Darus, and U. Kirmaci, "Refinements of Hadamard-type inequalities for quasiconvex functions with applications to trapezoidal formula and to special means," Comp. Math. Appl, vol. 59, pp. 225-232, 2010.

[3] R.-F. Bai, F. Qi, and B.-Y. Xi, "Hermite-Hadamard type inequalities for the $m$ - and $(\alpha, m)$ logarithmically convex functions," Filomat, vol. 27, no. 1, pp. 1-7, 2013.

[4] S.-P. Bai, S.-H. Wang, and F. Qi, "Some Hermite-Hadamard type inequalities for $n$-time differentiable $(\alpha, m)$-convex functions," J. Inequal. Appl, vol. 2012, no. 267, p. 11, 2012.

[5] S. Dragomir and R. Agarwal, "Two inequalities for differentiable mappings and applications to special means of real numbers and trapezoidal formula," Appl. Math. Lett, vol. 11, no. 5, pp. 91-95, 1998.

[6] D. Ion, "Some estimates on the Hermite-Hadamard inequality through quasi-convex functions," Annals of University of Craiova, Math. Comp. Sci. Ser, vol. 34, pp. 82-87, 2007.

[7] U. Kirmaci, "Inequalities for differentiable mappings and applications to special means of real numbers to midpoint formula," Appl. Math. Comput, vol. 147, no. 1, pp. 137-146, 2004.

[8] M. Latif, "New Hermite-Hadamard type integral inequalities for GA-convex functions with applications," Analysis (Munich), vol. 34, no. 4, pp. 379-389, 2014.

[9] M. Latif and S. Dragomir, "New inequalities of Hermite Hadamard type for $n$-times differentiable convex and concave functions with applications," Filomat, vol. 30, no. 10, pp. 2609-2621, 2016.

[10] C. Niculescu and L.-E. Persson, Convex Functions and their Applications. CMS Books in Mathematics: Springer-Verlag, 2005. 
[11] C. Pearce and J. Pečarić, "Inequalities for differentiable mappings with application to special means and quadrature formulae," Appl. Math. Lett, vol. 13, no. 2, pp. 51-55, 2000.

[12] F. Qi and B.-Y. Xi, "Some Hermite-Hadamard type inequalities for geometrically quasi-convex functions," Indian Acad. Sci. (Math. Sci.), vol. 24, no. 3, pp. 333-342, 2014.

[13] Y. Shuang, H.-P. Yin, and F. Qi, "Hermite-Hadamard type integral inequalities for geometricarithmetically s-convex functions," Analysis (Munich), vol. 33, no. 2, pp. 197-208, 2013.

[14] B.-Y. Xi, R.-F. Bai, and F. Qi, "Hermite-Hadamard type inequalities for the $m$ - and $(\alpha, m)$ geometrically convex functions," Aequationes Math, vol. 84, no. 3, pp. 261-269, 2012.

[15] B.-Y. Xi and F. Qi, "Hermite-Hadamard type inequalities for functions whose derivatives are of convexities," Nonlinear Funct. Anal. Appl, vol. 18, no. 2, pp. 163-176, 2013.

[16] T.-Y. Zhang, A.-P. Ji, and F. Qi, "On integral inequalities of Hermite-Hadamard type for $s$ geometrically convex functions," Abstr. Appl. Anal, vol. 2012, no. Article ID 560586, p. 14, 2012.

[17] T.-Y. Zhang, A.-P. Ji, and F. Qi, "Some inequalities of Hermite-Hadamard type for GA-convex functions with applications to means," Matematiche (Catania), vol. LXVIII, no. 1, p. 229-239, 2013.

Authors' addresses

M. A. Latif

University of Hail, Department of Mathematics, Hail 2440, Hail, Saudi Arabia

E-mail address: m_amer_latif@hotmail.com

Sever S. Dragomir

Mathematics, College of Engineering \& Science, Victoria University, PO Box 14428, Melbourne City, MC 8001, Australia

University of the Witwatersrand, School of Computer Science and Applied Mathematics, Private Bag 3, Wits 2050 Johannesburg, South Africa

E-mail address: sever.dragomir@vu.edu. au

\section{E. Momoniat}

University of the Witwatersrand, School of Computer Science and Applied Mathematics, Private Bag 3, Wits 2050 Johannesburg, South Africa

E-mail address: ebrahim.momoniatewits.ac.za 